\title{
STREET PHOTOGRAPHY AT SCALE: Photographically Documenting Neighborhoods
}

\section{EQUIPMENT}

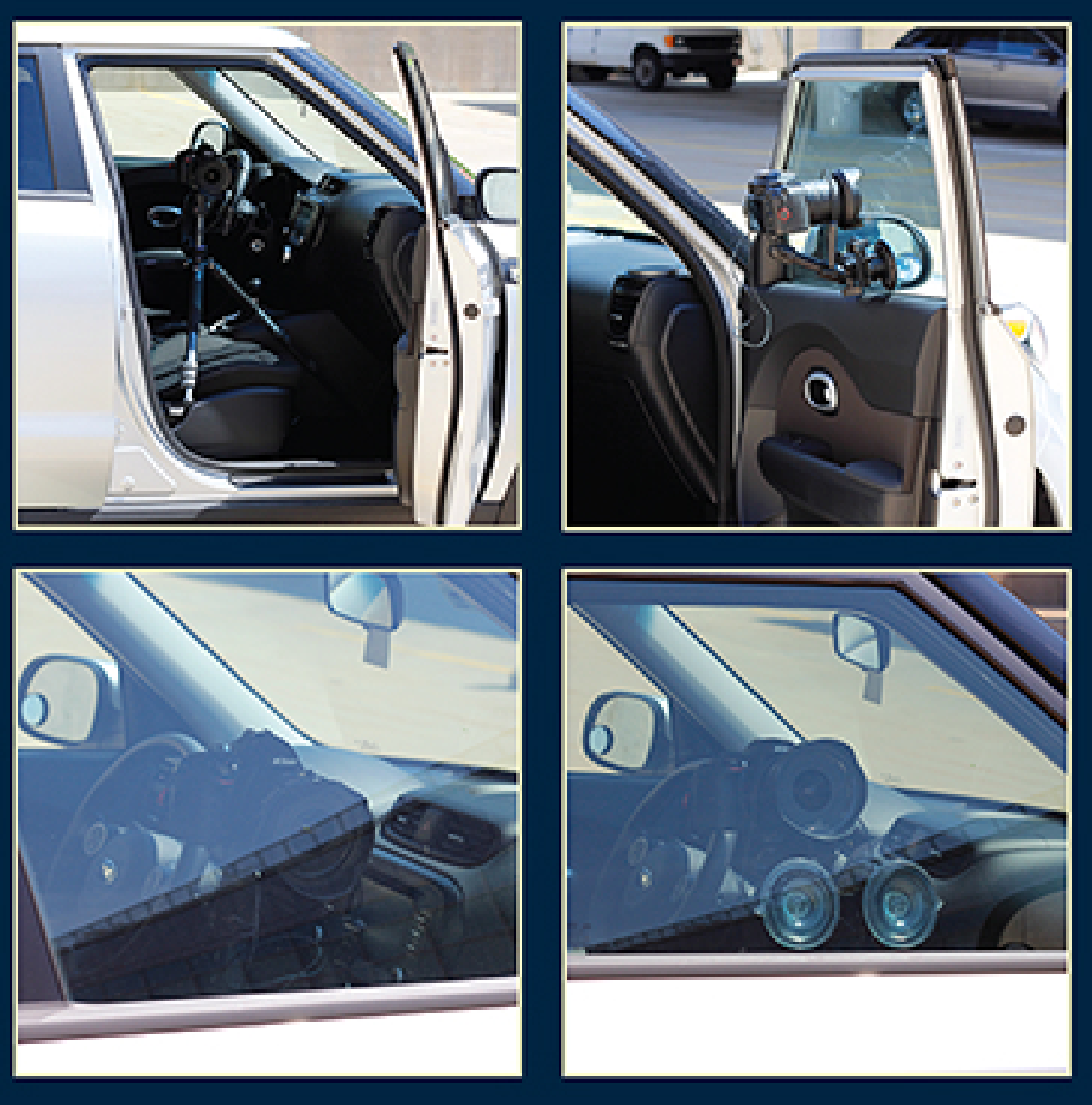

Mounting: Dolica LA600 Pro 60-inch Aluminum Alloy Traveler Edition (\$49.99, Amazon)

Mounting: Fat Gecko Camera and Camcorder Mount, Double (\$57.65, Amazon)

Camera: Nikon D3300 (\$413.84, Amazon)

Lens: Tamron 10-24 mm, f/3.5-4.5 Di II VC (\$499.00, Midwest Photo, Columbus, Ohio)

1 Storage: SanDisk Extreme PRO 64 GB SDXC Flash Memory Card (\$38.25, Amazon)

V Remote: Nikon 25395 MC-DC2

Remote Release Cord (\$35.70, Amazon)

Total cost with suction mount: \$1,044.44

The tripod was used initially in order test the effectiveness

of the project, but the suction mount provides a superior

outcome, with the camera closer to the glass, easier

set-up, and greater stability.

\section{OVERVIEW}

This project endeavors to create a workflow by which the built environment of a community can be photographically documented, more quickly than on foot, and without having to rely on services such as Google Street View, placing the intellectual control of the images in the control of the library or archive.

\section{GPS Metadata}

In order to make the images useful, context needs to be given for where the images were taken. In order to track GPS metadata, an app was installed on a mobile Android phone to record the GPS position of the car while moving.

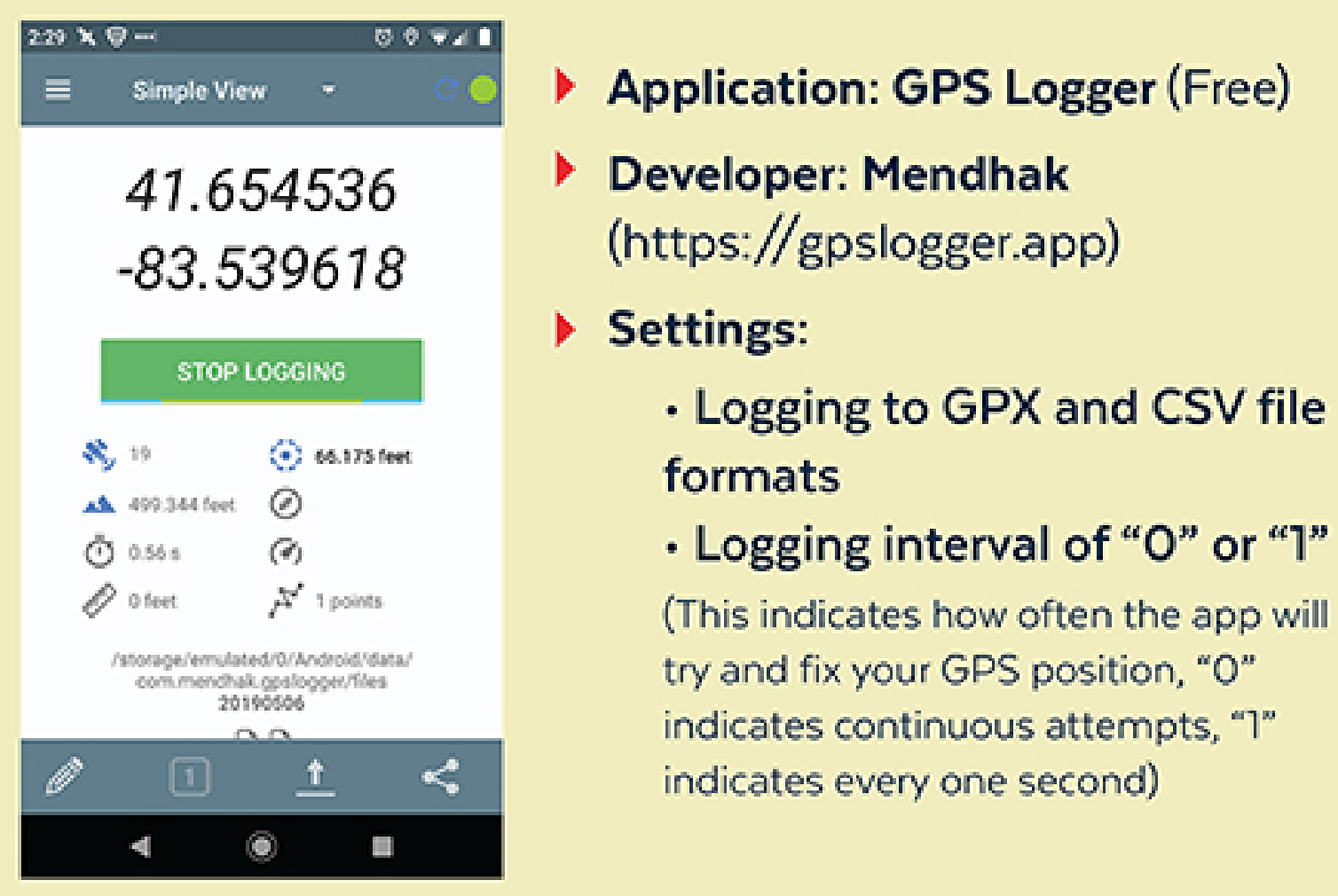

Note: Stand-alone GPS loggers are a possibility for increasing accuracy of readings, though it does mean an additional equipment cost.

\section{WORKFLOW}

Image Capture

- Camera is mounted in car using either tripod or suction mount

- Wide-angle lens is utilized to capture as much of the street as possible

Shutter speed is prioritized (1/1000 or faster) to eliminate motion blur

- Use remote to take lots of pictures quickly to keep eyes on the road when passing desired target (more pictures taken, the better chance of getting a good image)

Tip: Keep distance to target short in order to have quick auto-focus time

Image Metadata

- Synchronize camera clock to phone (or whatever is being used to gather GPS data), associating GPS metadata will be done by matching timestamps

- Area for development: automatically merging GPS data into embedded image metadata; manually

copying/pasting is slow and tedious

- Future plans: development of geospatial map for all digital assets for browsing, including images generated from this project

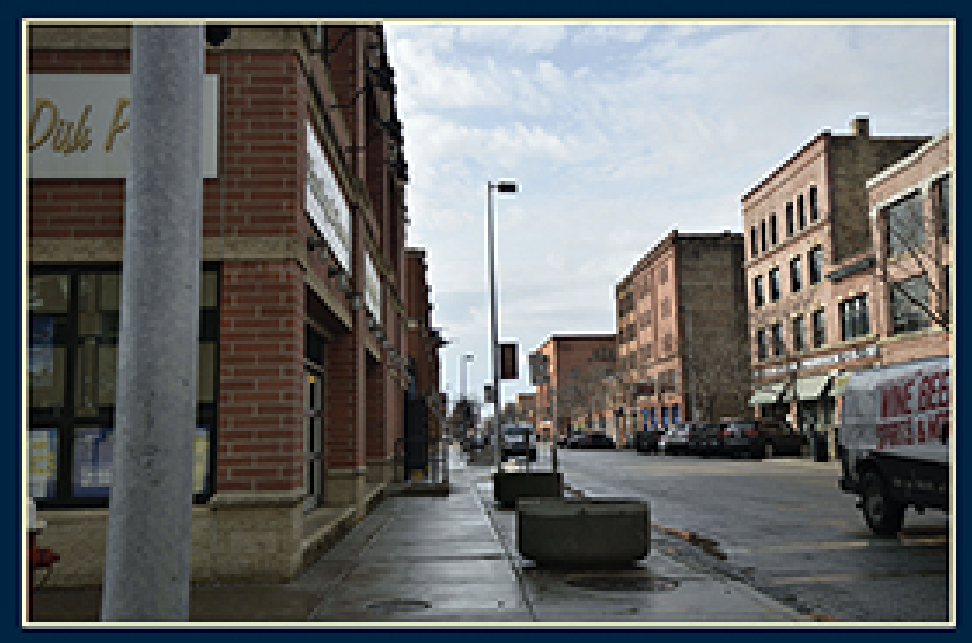

Society of Ohio Archivists, 2019 Meeting John Dewees, MLIS Supervisor Digitization Services Toledo Lucas County Public Library 